\title{
The Effects of Osteoarthritis on Quality of Life (QoL)
}

\author{
Qaim Ali*, Maheen Aftab, Sara Arshad \\ Department of Pharmacy, Ziauddin College of Pharmacy, Karachi, Pakistan.
}

\begin{abstract}
Objective: Functionality and quality of life in patients with osteoarthritis undergoing total arthroplasty of the knee.
Materials and Methods: This study was conducted in Ziauddin hospital, Karachi from April 2018 until August 2018, making up 40 individuals who have osteoarthrosis of the knee with an indication for total arthroplasty and not being carriers of any other disease affecting the limbs lower. Two scales of evaluation were applied at two different times: pre and postoperative. Health status questionnaire (SF-36) and Knee injury and osteoarthritis outcome score (KOOS) was applied and answered in the preoperative period (on the day of surgery) and the sixth postoperative week.
\end{abstract}

Results: Regarding the dimensions of activities of daily living and activities, sporting and leisure activities were not statistically significant. Our results go against the conclusions reported by Culliton [1], in a study of 13 patients submitted to TKA. The participants were evaluated before the arthroplasty and four weeks later and the results showed that there was a tendency for pain to decrease in that period.

Conclusion: It can be concluded that there was a tendency to decrease pain in this period accompanied by an improvement in the quality of life. Taking into account the results obtained, and stressing the concept that quality of life is related to the state of health of the person and that pain and functional limitation caused by OA negatively influence the well-being and the performance of the activities of the person's daily life, it can be concluded that the TCA contributes to the improvement of the performance of the person.

Keywords: Osteoarthritis, Joint, Arthroplasty, Articular cartilage, Osteoarthrosis, Knee injury, QoL.

\section{INTRODUCTION}

Osteoarthritis (OA) is the degeneration of articular cartilage resulting from a combination of mechanical, genetic, hormonal, bone, and metabolic factors [2]. Incurable, its prevalence has only increased over the years, with differences depending on the region of the world [3]. It is one of the main causes of disability around the world [4], being the main cause of physical limitations when reaching the age group above 60 years [5]. Due to longer life expectancy and a decline in fertility rates, the population over 60 is increasing more than other age groups [1]. With the growth of this population range, there is a need to promote the investigation of interventions to optimize their quality of life and health. One of the biggest causes for a high level of physical disability, reduced capacities in activities of daily living and high economic costs with health for the elderly is the presence of OA [6], which negatively impacts work, activities of daily life and in the relationship life of these individuals, especially due to high levels of pain [7] and due to impaired balance [8].

The most widely accepted general recommendations on OA treatment are low-impact activities for the affected joint, with aerobic and / or strengthening exercises practiced on the ground or in an aquatic environment [9]. The joints commonly affected are the hip, spine, hand, ankle, and knee [10]. The improvement in strength and proprioception of the muscles around the joint contributes to the improvement of neural

*Address correspondence to this author at the Department of Pharmacy, Ziauddin College of Pharmacy, Karachi, Pakistan.

E-mail: qaimali59@gmail.com activation, the quality of cartilage and intra-coordination, and intermuscular, thus optimizing the load-absorbing mechanism of the knee and hip during gait [3]. Among the physiotherapeutic approaches used to treat OA (resistance ground exercises, aquatic exercises, thermal agents, taping, manual therapies, and tai chi) [11], the method developed by Sir John Charnley, worldwide known as arthroplasty, it was selected for this investigation because it is a treatment which is effective with physical benefits eliminating the discomfort and adding to the Quality of Life (QoL) of patients [12].

Knee arthroplasty was performed for the first time in 1968 and is one of the procedures most successful in all medicine [13]. Arthrodesis eliminates pain and restores stability to the knee, but this surgery imposes great functional limitations on the patient and becomes practically unviable when the involvement is bilateral. The first arthroplasty attempts at the knee appeared at the end of the last century, with the techniques of interposition and resection [2], with results almost always compromised by infection, ankylosis, and instability. After the introduction of hinge arthroplasty [14] and hemiarthroplasties [9], research was intensified. In 1970, Gunston presented the study of polycentric arthroplasty, cemented with methyl methacrylate, and obeying the principle of low friction (metal on high-density polyethylene). Thus, the purpose of this study was to evaluate whether arthroplasty improves the quality of life and functionality of patients with knee osteoarthritis undergoing the total arthroplasty. In this regard, the following hypothesis is proposed for this study. 


\section{Hypothesis}

This study hypothesizes that total arthroplasty can improve the quality of life and functionality of patients with knee OA, with decreased pain and improvement of dynamic balance.

\section{Aims and Objectives}

\section{Primary Objective}

To evaluate the incapacity generated by knee problems and the quality of life in patients with knee osteoarthritis undergoing total arthroplasty.

\section{Secondary Objectives}

Based on the primary objective, the secondary objectives that guide this work are:

- To characterise the patients according to the socio-demographic variables gender, age, Body Mass, municipality of origin and profession;

- To compare the results obtained in the preoperative period and six weeks after surgery regarding the quality of life of patients with osteoarthritis of the knee submitted to total arthroplasty;

- To compare the results obtained in the preoperative period and six weeks after surgery regarding the degree of functionality of patients with osteoarthritis of the knee underwent total arthroplasty;

\section{MATERIALS AND METHODS}

The present work is a study of quantitative nature and character longitudinal perspective since it aims to describe the variations occurring in them individuals over six weeks, allowing the acquisition and deepening of the subject and the variables under study. The longitudinal drawings are characterised by the existence of a follow-up or follow-up, in which a temporal sequence, interventions therapeutic or an evolutionary fact are studied [15]. This study was conducted in Ziauddin hospital, Karachi from April 2018 until August 2018, making up 40 individuals who have osteoarthrosis of the knee with an indication for total arthroplasty and not being carriers of any other disease affecting the limbs lower. Two scales of evaluation will be applied at two different times: pre and postoperative. The validation of the following instruments: Health status questionnaire (SF-36) and Knee injury and osteoarthritis outcome score (KOOS) that will be applied and answered in the preoperative period (on the day of surgery) and the sixth postoperative week. Knee injury and osteoarthritis outcome score (KOOS) is a specific questionnaire directed to knee disorders that assesses pain, physical function, and quality of life. The SF-36 questionnaire is used to investigate the quality of life and is structured in 36 items.

\section{RESULTS}

\section{Characterization of Participants}

The sample under study consisted of 40 female users (62.5\%). Concerning the profession, it is observed that the retirement situation was predominant in $80 \%$ of cases. The remaining $20 \%$ of users were still active in working in various professions (Table 1).

Table 1. Categorization of Participants according to Gender and Occupation.

\begin{tabular}{|l|c|c|}
\hline Variables & \multicolumn{2}{|c|}{ Nample } \\
\hline \multicolumn{2}{|l|}{} \\
\hline Gender & 25 & 62.5 \\
\hline Female & 15 & 37.5 \\
\hline Male & $\mathbf{4 0}$ & $\mathbf{1 0 0 . 0}$ \\
\hline TOTAL & 32 & 80.0 \\
\hline Profession & 2 & 5.0 \\
\hline Reformed & 1 & 2.5 \\
\hline Domestic & 1 & 2.5 \\
\hline Farmer & 1 & 2.5 \\
\hline Merchant & 1 & 2.5 \\
\hline Nurse & 1 & 2.5 \\
\hline Pharmacist & 1 & 2.5 \\
\hline Government Employee & $\mathbf{4 0}$ & $\mathbf{1 0 0 . 0}$ \\
\hline Factory Worker & & \\
\hline TOTAL & & \\
\hline
\end{tabular}

Participants had a mean age of 72 years $(71.6 \pm 8.1)$, statistically significant variations between women and men. Regarding the BMI we obtained an average value of $27.2( \pm 3.9)$ for the total sample, without significant differences between sexes (Table 2).

Table 2. Descriptive Statistics Obtained for the Variables: Age and Mass Index Body, by Gender and for the Total Sample.

\begin{tabular}{|l|c|c|c|c|}
\hline Variables & $\begin{array}{c}\text { Women } \\
(\mathbf{n = 2 5})\end{array}$ & $\begin{array}{c}\text { Men } \\
(\mathbf{n = 1 5})\end{array}$ & $\begin{array}{c}\text { Sample } \\
(\mathbf{n = 4 0 )}\end{array}$ & $\mathbf{p}$ \\
\hline Age $(\mathrm{M} \pm \mathrm{SD})$ & $71.2 \pm 8.7$ & $72.3 \pm 7.3$ & $71.6 \pm 8.1$ & 0.270 \\
BMI $(\mathrm{M} \pm \mathrm{SD})$ & $26.7 \pm 4.0$ & $28.0 \pm 3.8$ & $27.2 \pm 3.9$ & 0.675 \\
\hline
\end{tabular}

M-Mean; SD-Standard Deviation; BMI- Index of Body mass.

\section{Analysis of Quality of Life Assessed by SF-36 v2}

The following Table 3 sets out the descriptive statistics obtained in the various dimensions of the SF-36 v2 at the first evaluation (M1), before surgery. The descriptive analysis shows that the dimensions of quality averages of life, less than 
50 were body pain (BD) (42.2) and physical function (PF) $(48,8)$. On the other hand, general health (GS) $(61.9)$ was the component in which a higher quality of life. Observing the dispersion measures (standard deviation and variation) it is verified that the largest. The variability of values obtained was in the emotional performance dimension (EP).

Table 3. Descriptive Statistics Obtained in the Different Dimensions of SF-36 v2 when First Evaluated.

\begin{tabular}{|l|c|c|c|c|}
\hline SF-36 v2 & Minimal & Maximum & M \pm SD \\
$48.8 \pm 7.0$ & V \\
Dimensions & 40.0 & 66.7 & 9.3 \\
$\begin{array}{l}\text { Physical } \\
\text { Function (PF) }\end{array}$ & 25.0 & 80.0 & $52.5 \pm 11.0$ & 121.8 \\
\hline $\begin{array}{l}\text { Physical } \\
\text { Performance (PP) }\end{array}$ & 16.7 & 65.4 & $42.2 \pm 12.4$ & 153.6 \\
\hline Body Pain (BP) & 40.0 & 81.6 & $61.9 \pm 11.2$ & 124.6 \\
\hline General Health (GH) & 40.0 & 75.0 & $58.9 \pm 7.2$ & 51.9 \\
\hline Vitality (VT) & 30.0 & 80.0 & $54.3 \pm 13.4$ & 178.9 \\
\hline Social Role (SR) & 30.0 & 80.0 & $54.5 \pm 14.0$ & 194.6 \\
\hline $\begin{array}{l}\text { Emotional } \\
\text { Performance (EP) }\end{array}$ & 40.0 & 72.0 & $55.3 \pm 11.0$ & 121.3 \\
\hline Mental Health (MH) & & & & \\
\hline
\end{tabular}

M1- First moment of evaluation; M-Mean; SD-Standard Deviation; V-Variance.

Observing the mean values obtained in the various dimensions of quality of life in M2, the fact that in any component there has been an average lower than the reference value 50 . Thus, it can be seen in Table $\mathbf{4}$ that the less-quoted dimensions were in mean terms, physical function (PF) (53.6), and physical performance (PP) (58.4). On the other hand, the highest mean values of quality of life in M2 were body pain components (BP) (71.6).

Table 4. Descriptive Statistics obtained in the Different Dimensions of the SF-36 v2 when Second Evaluation.

\begin{tabular}{|l|c|c|c|c|}
\hline SF-36 v2 & \multicolumn{4}{|c|}{ M2 } \\
\hline $\begin{array}{l}\text { Dimensions } \\
\text { Physical } \\
\text { Function (PF) }\end{array}$ & $\begin{array}{c}\text { Minimal } \\
\text { Maximum } \\
78.5\end{array}$ & $\begin{array}{c}\text { M } \pm \text { SD } \\
53.6 \pm 11.1\end{array}$ & $\begin{array}{c}\text { V } \\
122.4\end{array}$ \\
\hline $\begin{array}{l}\text { Physical } \\
\text { Performance (PP) }\end{array}$ & 30.0 & 80.0 & $58.4 \pm 11.2$ & 126.1 \\
\hline Body Pain (BP) & 39.6 & 95.0 & $71.6 \pm 12.0$ & 143.5 \\
\hline General Health (GH) & 50.0 & 83.6 & $67.8 \pm 10.0$ & 99.9 \\
\hline Vitality (VT) & 43.0 & 78.0 & $62.0 \pm 8.3$ & 68.2 \\
\hline Social Role (SR) & 48.0 & 88.0 & $69.2 \pm 9.7$ & 93.3 \\
\hline $\begin{array}{l}\text { Emotional } \\
\text { Performance (EP) }\end{array}$ & 36.0 & 86.0 & $66.5 \pm 12.6$ & 158.7 \\
\hline Mental Health (MH) & 48.0 & 76.0 & $64,0 \pm 7,5$ & 55.7 \\
\hline
\end{tabular}

M2- Second moment of evaluation; $M$ - Mean; SD-Standard Deviation; V-Variance.

Table 5 compares the means obtained in the physical component of SF-36 v2 (PF, PP, BP, and HG) at the two evaluated moments. As indicated, there were statistically significant improvements $(p<0.05)$ in all dimensions of the physical component to the second evaluation moment.

Table 5. Means Obtained in the Two Moments of Evaluation for the Dimension Physical Function.

\begin{tabular}{|c|c|c|c|}
\hline & $\begin{array}{c}\text { M1 } \\
(\mathbf{M} \pm \text { SD) }\end{array}$ & $\begin{array}{c}\text { M2 } \\
(\mathbf{M} \pm \text { SD) }\end{array}$ & $p$ \\
\hline PF & $48.8 \pm 7.0$ & $53.6 \pm 11.1$ & 0.005 \\
\hline PP & $52.5 \pm 11.0$ & $58.4 \pm 11.2$ & 0.001 \\
\hline BP & $42.2 \pm 12.4$ & $71.6 \pm 12.0$ & $<0.001$ \\
\hline HG & $61.9 \pm 11.2$ & $67.8 \pm 10.0$ & $<0.001$ \\
\hline
\end{tabular}

M1-Moment 1, M2-Moment 2, PF-Physical function, PP-Physical performance, BPBody Pain, HG-Health in general, $p$-Significance t-test for paired samples.

Table 6 reveals the mental component of SF-36 v2 (PF, PP, $\mathrm{BP}$, and $\mathrm{HG}$ ), and analyzing the variations in the means recorded in M1 and M2 using the sample t-test paired (Table $10)$, we concluded by a significant improvement $(p<0.05)$ in quality of life in all dimensions, except for vitality (VT) whose is not statistically relevant $(\mathrm{p}=0.062)$.

Table 6. Averages Obtained in the Two Moments of Evaluation for the Dimension Vitality.

\begin{tabular}{|l|c|c|c|}
\hline & $\begin{array}{c}\text { M1 } \\
(\mathbf{M} \pm \text { SD })\end{array}$ & $\begin{array}{c}\text { M2 } \\
(\mathbf{M} \pm \text { SD) }\end{array}$ & $p$ \\
\hline VT & $58.9 \pm 7.2$ & $62.0 \pm 8.3$ & 0.062 \\
\hline SF & $54.3 \pm 13.4$ & $69.2 \pm 9.7$ & $<0.001$ \\
\hline PE & $54.5 \pm 14.0$ & $66.5 \pm 12.6$ & $<0.001$ \\
\hline MH & $55.3 \pm 11.0$ & $64.0 \pm 7.5$ & $<0.001$ \\
\hline
\end{tabular}

M1-Moment 1, M2-Moment 2, VT-Vitality, SF-Social function, PE-Performance emotional, $M H$-Mental health, p-Significance t-test for paired samples.

\section{The Function of the Knee (KOOS Analysis)}

In the following tables, we present the data obtained in the function of the knee through the KOOS analysis. Observing the averages obtained during the first evaluation, it is verified that the less marked was the one related to sport and physical and leisure activity (13.6 \pm 2.9$)$. Already $(62.4+/$ - 15.4) were observed in the most the highest standard deviation, which means that the variability in the responses to these issues was great (Table 7).

Table 7. Descriptive Statistics Obtained in the Different Dimensions of KOOS when First Evaluated.

\begin{tabular}{|c|c|c|c|c|}
\hline KOOS & \multicolumn{4}{|c|}{ M1 } \\
\hline Dimensions & Minimum & Maximum & $\mathbf{M} \pm \mathbf{S D}$ & $\boldsymbol{V}$ \\
\hline Symptoms (S) & 31.9 & 96.1 & $62.4 \pm 15.4$ & 238.2 \\
\hline Pain $(\mathrm{P})$ & 34.2 & 87.0 & $58.6 \pm 12.5$ & 157.3 \\
\hline Life Activities (LA) & 32.8 & 88.7 & $61.7 \pm 13.2$ & 174.2 \\
\hline Sports/Leisure Activities (S/LA) & 11.0 & 21.0 & $13.6 \pm 2.9$ & 9.0 \\
\hline Quality of Life (QoL) & 34.8 & 78.5 & $43.8 \pm 11.4$ & 129.3 \\
\hline
\end{tabular}

MI- First moment of evaluation; M- Mean; SD-Standard Deviation; V-Variance. 
In the second moment of evaluation (Table 8), it was verified that the subscale sport/physical activity and leisure activities continued to be, on average, less marked $(13.9 \pm 11.4)$. At the other extreme, the highest functional average was recorded in the concerning pain.

Table 8. Descriptive Statistics Obtained in the Different Dimensions of KOOS when the Second Evaluation was Made.

\begin{tabular}{|c|c|c|c|c|}
\hline KOOS & & M2 & & \\
\hline Dimensions & Minimum & Maximum & $\mathbf{M} \pm \mathbf{S D}$ & $\mathbf{V}$ \\
\hline Symptoms (S) & 50.6 & 89.4 & $66.5 \pm 11.9$ & 141.8 \\
\hline Pain (P) & 50.6 & 98.0 & $71.8 \pm 15.6$ & 243.0 \\
\hline Life Activities (LA) & 50.0 & 86.2 & $64.0 \pm 11.1$ & 123.7 \\
\hline Sports/Leisure Activities (S/LA) & 5.0 & 60.0 & $13.9 \pm 11.4$ & 128.8 \\
\hline Quality of Life (QoL) & 29.5 & 73.3 & $49.9 \pm 11.4$ & 129.8 \\
\hline
\end{tabular}

M2-First moment of evaluation; M- Mean; SD-Standard Deviation; V-Variance.

The comparison between the mean values of the first and second statistical significance in the symptoms, pain, and quality of life dimensions. Already the difference of averages observed between activities of daily living and sports or non-leisure activities was statistically significant by the t-test for paired samples (Table 9).

Table 9. Comparison of the Means Obtained in the Two Evaluation Moments in the KOOS Questionnaire.

\begin{tabular}{|c|c|c|c|}
\hline KOOS & M1 & M2 & p \\
\hline & $(\mathbf{M} \pm$ SD $)$ & $(\mathbf{M} \pm$ SD $)$ & \\
\hline S & $62.4 \pm 15.4$ & $66.5 \pm 11.9$ & 0.015 \\
\hline P & $58.6 \pm 12.5$ & $71.8 \pm 15.6$ & $<0.001$ \\
\hline SA & $61.7 \pm 13.2$ & $64.0 \pm 11.1$ & 0.060 \\
\hline QoL & $13.6 \pm 3.0$ & $13.9 \pm 11.4$ & 0.885 \\
\hline
\end{tabular}

M1- Moment 1, M2-Moment 2, S- Symptoms, P-Pain, LA-Daily life activities, S/LASport/leisure activities, QoL-Quality of life, $p$-Significance t-test for paired samples.

Table 10 correlates the scores obtained between the same dimension, in M1 and M2. There are significant correlations between these dimensions, which may indicate, for example, those users who presented good functionality in the life activities preoperative period also present it in the second evaluation $(r=0.823)$.

Table 10. Correlations Obtained between the Various Dimensions of KOOS in the Two Moments Evaluated.

\begin{tabular}{|c|c|c|c|c|c|}
\hline & $\mathbf{S}^{\mathrm{M} 1}$ & $\mathbf{P}^{\mathrm{M} 1}$ & $\mathbf{L A}^{\mathrm{M} 1}$ & $\mathbf{S} / \mathbf{L A}^{\mathrm{M}}$ & QoL $^{\mathrm{M1}}$ \\
\hline $\mathbf{S}^{\mathbf{M} 2}$ & $0.760 *$ & & & & \\
\hline $\mathbf{P}^{\mathrm{M} 2}$ & & $0.683^{*}$ & & & \\
\hline $\mathbf{L A}^{\mathrm{M} 2}$ & & & $0.823 *$ & & \\
\hline $\mathrm{S} / \mathbf{L} \mathbf{A}^{\mathrm{M} 2}$ & & & & 0.297 & \\
\hline $\mathbf{Q o L}^{\mathrm{M} 2}$ & & & & & $0.694 *$ \\
\hline
\end{tabular}

* The correlation is significant at the level of 0.01 (bi-flow).

M1-Moment 1, M2-Moment 2, S-Symptoms, P-Pain, LA-Daily life activities, S/LASport/leisure activities, QoL-Quality of life, $p$-Significance t-test for paired samples.

\section{DISCUSSION}

The first topic of discussion refers to the fact that the sample studied mainly by female members, with a percentage of $62.5 \%$. It is an occurrence that agrees with the literature that refers to a higher prevalence of OA in females [16]. These results also go to the study by [6] who evaluated clinically and epidemiologically $\mathrm{OA}$ in 84 institutionalised patients and concluded that the majority were female, with $70.9 \%$ of the cases [17]. Such findings are probably due to postmenopausal osteoporosis, physical activity levels during the active life and to the fact that the majority of the women present a muscular massless developed compared to men [10].

Regarding the profession, it is observed that the retirement situation is predominant in $80 \%$ of cases. Being the retirement age in Pakistan of 66 years and 3 months, this related to the mean age of the participants who are 72 years $(71.6 \pm 8.1)$, results similar to the mean ages of other studies of this genre in which the mean was of 74.1 years and in the age groups between 70 and 79 years old, 60 to 70 years [18]. However, the study does not observe statistically significant variations between women and men. The age, not being considered a mandatory factor for diagnosis of $\mathrm{OA}$, it is considered as one of the risk factors for the development of the disease.

Concerning BMI, an average value of $27.2( \pm 3.9)$ was obtained $(\mathrm{BMI}=$ pre-obese value) for the total sample. Being overweight is considered one of the risk factors for the development of OA of the knee since it generates an increase of pressure on the articulation. The results obtained are in agreement with the literature and with the findings published by Giggins [11] in a study of 40 patients that allowed the conclusion that the number of obese patients with OA is superior in comparison to normal-weight patients.

The analysis of the variables of QoL of the patients in the two moments of evaluation (pre and postoperative period) was performed using the MOS questionnaire SF-36 v2.

The results obtained for this the first and second assessments show responses in the self-perception of health during the second evaluation, where $55.0 \%$ of respondents indicated that their health was good, compared to $35 \%$ obtained in the first evaluation. A study by Finch [14] to 2135 people (58.6\% women and mean age of 69.4 years), based on data from the Health Project, Welfare and Aging in Latin America and the Caribbean (SABE) on the determinants of self-perception in health concluded that the presence of chronic diseases is related to a worse self-perception of health [19].

This data goes against those obtained in our investigation because the patients with OA more favorable health perception after surgery compared to the disease (first evaluation). These results are probably explained by the fact that hospitals such as Ziauddin Hospital are capable of reducing painful 
complaints through surgery, improving the amplitude articulate, improve walking capacity, and ambulation. Already in 1993, this surgery presented satisfactory results. Afterward de Rooij [12] studied 40 patients submitted to total arthroplasty, they were able to conclude that the intervention was shown to be a satisfactory method in cases of rheumatoid arthritis and osteoarthrosis, elimination of pain, correction of deformities and instabilities, and obtaining good functional capacity.

In the current study, the analysis of the functional perception/knee problems variable was performed through the application of the Knee injury and osteoarthritis questionnaire outcome score (KOOS), which evaluates five dimensions: extra symptomatology to pain $(\mathrm{S})$, pain $(\mathrm{P})$, activities of daily living (LA), sports and leisure activities (S/LA) and quality of life-related to the knee (QoL). The comparison between the mean values of the first and second $(\mathrm{p}=0.015)$, pain ( $\mathrm{p}$ $<0.001)$, and quality of life $(p<0.001)$. Regarding the dimensions of activities of daily living and activities, sporting and leisure activities were not statistically significant. Our results go against the conclusions reported by Culliton [1], in a study of 13 patients submitted to TKA. The participants were evaluated before the arthroplasty and four weeks later and the results showed that there was a tendency for pain to decrease in that period.

Another work that goes against these results is the study developed by Conchie [20] in their study developed with 39 patients who underwent TKA, who were evaluated at three different times (1, 3 and 6 months after surgery) through measures of functional performance, namely using the 6-minute walk test, Timed Up and Go and KOOS questionnaire (Knee and Osteoarthritis Outcome Score). As for the results of $\mathrm{KOOS}$, the concluded that there was an increase in the functionality of the knee, in all moments of the follow-up, except for the subscale related to the activities sporting and leisure activities, whose improvement was only noticeable from the evaluation of the 3 months.

\section{CONCLUSION}

It can be concluded that there was a tendency to decrease pain in this period accompanied by an improvement in the quality of life. Taking into account the results obtained, and stressing the concept that quality of life is related to the state of health of the person and that pain and functional limitation caused by OA negatively influence the well-being and the performance of the activities of the person's daily life, it can be concluded that the TCA contributes to the improvement of the performance of the person. It was also observed that the TKA, besides showing improvement in physical performance, also contributed in a very significant way to the improvement of the participants' emotional and social activity. For the accomplishment of this work, all the patients with the diagnosis of OA submitted to TKA during the period defined for the study.

\section{CONFLICT OF INTEREST}

Declared none.

\section{ACKNOWLEDGEMENTS}

Declared none.

\section{AUTHORS' CONTRIBUTION}

All authors have equally contributed.

\section{REFERENCES}

[1] Culliton SE, Bryant DM, Overend TJ, MacDonald SJ, Chesworth BM. The relationship between expectations and satisfaction in patients undergoing primary total knee arthroplasty J Arthroplast 2012; 27(3): 490-2.

DOI: $10.1016 /$ j.arth.2011.10.005

[2] Almangoush A, Herrington L, Attia I, Jones R, Aldawoudy A, Aziz AA, Waley A. Cross-cultural adaptation, reliability, internal consistency and validation of the Arabic version of the Knee injury and Osteoarthritis Outcome Score (KOOS) for Egyptian people with knee injuries. Osteoarthritis Cartilage 2013; 21(12): 1855-64. DOI: 10.1016/j.joca.2013.09.010

[3] Berth A, Pap G. Stemless shoulder prosthesis versus conventional anatomic shoulder prosthesis in patients with osteoarthritis. J Orthopaed Traumatol 2013; 14(1): 31-7.

DOI: $10.1007 / \mathrm{s} 10195-012-0216-9$

[4] Chen AGCM, Gupte C, Akhtar K, Smith P, Cobb J. The global economic cost of osteoarthritis: How the UK compares. Arthritis 2012; 2012: Article ID 698709.

DOI: $10.1155 / 2012 / 698709$

[5] Beswick AD, Wylde V, Gooberman-Hill R, Blom A, Dieppe P. What proportion of patients report long-term pain after total hip or knee replacement for osteoarthritis? A systematic review of prospective studies in unselected patients. BMJ Open 2012; 2(1): e000435.

DOI: 10.1136/bmjopen-2011-000435

[6] Gouttebarge V, Inklaar H, Backx F, Kerkhoffs G. Prevalence of osteoarthritis in former elite athletes: A systematic overview of the recent literature. Rheumatol Int 2015; 35(3): 405-18. DOI: 10.1007/s00296-014-3093-0

[7] Bruyère $\mathrm{O}$, Ethgen $\mathrm{O}$, Neuprez $\mathrm{A}$, et al. Health-related quality of life after total knee or hip replacement for osteoarthritis: A 7-year prospective study. Arch Orthop Trauma Surg 2012; 132(11): 1583-7. DOI: $10.1007 / \mathrm{s} 00402-012-1583-7$

[8] Bergin SM, Munteanu SE, Zammit GV, Nikolopoulos N, Menz HB. Impact of first metatarsophalangeal joint osteoarthritis on health-related quality of life. Arthritis Care Res 2012; 64(11): 1691-8. DOI: 10.1002/acr.21729

[9] Brand CA, Harrison C, Tropea J, Hinman RS, Britt H, Bennell K. Management of osteoarthritis in general practice in Australia. Arthritis Care Res 2014; 66(4): 551-8. 
DOI: $10.1002 /$ acr.22197

[10] Gouttebarge V, Aoki H, Kerkhoffs G. Prevalence and determinants of symptoms related to mental disorders in retired male professional footballers. J Sports Med Phys Fitness 2015; 56(5): 648-54.

[11] Giggins OM, Fullen BM, Coughlan GF. Neuromuscular electrical stimulation in the treatment of knee osteoarthritis: A systematic review and meta-analysis. Clin Rehabil 2012; 26(10): 867-81. DOI: $10.1177 / 0269215511431902$

[12] de Rooij M, Steultjens MP, Avezaat E, et al. Restrictions and contraindications for exercise therapy in patients with hip and knee osteoarthritis and comorbidity. Phys Therap Rev 2013; 18(2): 101-11. DOI: 10.1179/1743288X12Y.0000000056

[13] Bossen D, Buskermolen M, Veenhof C, de Bakker D, Dekker J. Adherence to a web-based physical activity intervention for patients with knee and/or hip osteoarthritis: A mixed method study. J Med Int Res 2013; 15(10): e223.

DOI: $10.2196 /$ jmir.2742

[14] Finch CF, Kemp JL, Clapperton AJ. The incidence and burden of hospital-treated sports-related injury in people aged $15+$ years in Victoria, Australia, 2004-2010: A future epidemic of osteoarthritis? Osteoarthritis Cartilage 2015; 23(7): 1138-43. DOI: 10.1016/j.joca.2015.02.165

[15] Flick U. Introducing research methodology: A beginner's guide to doing a research project. Germany: Sage 2015; Available at: https://uk.sagepub.com/en-gb/eur/introducing-research-methodo- $\log$ /book241565

[16] Hart HF, Collins NJ, Ackland DC, Crossley KM. Is impaired knee confidence related to worse kinesiophobia, symptoms, and physical function in people with knee osteoarthritis after anterior cruciate ligament reconstruction? J Sci Med Sport 2015; 18(5): 512-7. DOI: 10.1016/j.jsams.2014.09.011

[17] Graven-Nielsen T, Wodehouse T, Langford RM, Arendt-Nielsen L, Kidd BL. Normalization of widespread hyperesthesia and facilitated spatial summation of deep-tissue pain in knee osteoarthritis patients after knee replacement. Arthritis Rheum 2012; 64(9): 2907-16. DOI: 10.1002/art.34466

[18] Girbés EL, Meeus M, Baert I, Nijs J. Balancing "hands-on" with "hands-off" physical therapy interventions for the treatment of central sensitization pain in osteoarthritis. Manual Therapy 2015; 20(2): 349-52.

DOI: $10.1016 /$ j.math.2014.07.017

[19] Epinette JA, Brunschweiler B, Mertl P, Mole D, Cazenave A, French Society of Hip and Knee. Unicompartmental knee arthroplasty modes of failure: wear is not the main reason for failure: A multicentre study of 418 failed knees. Orthop Traumatol Surg Res 2012; 98(6): S124-S30.

DOI: $10.1016 /$ j.otsr.2012.07.002

[20] Conchie H, Clark D, Metcalfe A, Eldridge J, Whitehouse M. Adolescent knee pain and patellar dislocations are associated with patellofemoral osteoarthritis in adulthood: A case control study. Knee 2016; 23(4): 708-11. DOI: 10.1016/j.knee.2016.04.009 